\title{
Predictive risk factors for post-traumatic stress symptoms among nurses during the Italian acute COVID-19 outbreak
}

\begin{abstract}
BACKGROUND
The aim of the study was to investigate the posttraumatic stress disorder risk in nurses, detecting the relationship between distress experience and personality dimensions in the Italian acute COVID-19 outbreak. The study is an observational study conducted in March 2020.
\end{abstract}

\section{PARTICIPANTS AND PROCEDURE}

Mental screening was carried out in the Laboratory of Clinical Psychology on $N=36$ nurses in the age range 22-64 years $(M=37.30, S D=12.60) .76 .3 \%$ were working in nursing care with confirmed COVID-19 patients; $47.4 \%$ of nurses worked in a high COVID-19 rate environment, whereas $52.6 \%$ worked in a low COVID-19 rate environment.

\section{RESULTS}

The results confirm relation between anxiety and peritraumatic dissociation and posttraumatic stress; also anxiety is positively correlated with the agreeableness variable. Our finding was obtained in an acute Italian COVID-19 outbreak and measured and quantified the psychological response of nurses in terms of anxiety as an early reaction for emotional distress and high risk for posttraumatic stress disorders; the personality dimensions did not mediate the emotional distress or the probable risk for posttraumatic stress disorder. Nurses appeared to be exposed to mental distress and needed help.

\section{CONCLUSIONS}

The results evidenced the need to carry out a mental health program for health workers (especially nursing professionals).

KEY WORDS

clinical psychology; pandemic; COVID-19; emotional distress; mental health program 


\section{BACKGROUND}

The current COVID-19 pandemic is affecting the mental health of the population. Some studies started to detect the risk for the mental health of health care workers (Rossi et al., 2020; Lai et al., 2020). According to those studies, health workers appeared to be exposed to a high risk for depression, anxiety, and mental issues as a response to the acute COVID-19 outbreak, particularly among young women and frontline healthcare workers. Most research conducted in the coronavirus pandemic has been conducted by applying a survey method trying to involve a large number of health care workers, as well the general population to have preliminary data about the impact of the pandemic on mental health; they screened mental health in the population, detecting general mental suffering. The limitation of those studies is in the application of survey methodology: it is evident there lacks a gold standard for the psychological evaluation setting, so the results could be exposed to a high risk of unreliability.

The next investigations should be more detailed and reliable than currently, revealing the psychopathological impact on mental health in health workers by applying the gold standard for mental health measurement and study design application.

The February-March 2020 period was the Italian acute COVID-19 outbreak and stressed the healthcare system in terms of management of hospitalization procedures and management of the emotional impact on professionals in hospital emergency worried about the risk for their own health.

According to Lai et al.'s (2020) findings and following other recent studies based on an online survey detecting the negative impact of the pandemic (Rossi et al., 2020), we wanted to evaluate the mental health of nurses in the Italian acute COVID-19 outbreak, applying psychological measurements for posttraumatic disorder risk in a traditional psychological setting.

The study aimed to investigate the posttraumatic stress disorder risk in the most fragile targets among health care workers (female nurses), detecting the relationship between distress experience and personality dimensions.

\section{PARTICIPANTS AND PROCEDURE}

\section{STUDY DESIGN}

Participants were enrolled in the Clinical Psychology Laboratory of the University of L'Aquila. Informed consent was obtained from each participant at the time of enrolment and the study adhered to the Declaration of Helsinki.

The study is an observational study based on mental screening conducted in March 2020. During this period, the total confirmed cases of COVID-19 exceeded 15000 in Italy. Trained clinical psychologists, blind to the objectives of the study, conducted the psychological screening in a quiet, dedicated room. The duration of the evaluations was 45 minutes. Data were collected anonymously.

\section{PARTICIPANTS}

Eligible participants were female nurses aged 22-64 years $(M=37.30, S D=12.60)$. Demographic characteristics of the $N=36$ participants are: $60.5 \%$ ( $n=23$ ) of them were married, $71 \%$ having no children, $76.3 \%$ working in nursing care with confirmed COVID-19 patients (named frontline; second-line nurses have been identified as nursing care working with infectious patients but no confirmed COVID-19); $47.4 \%$ of nurses worked in a high COVID-19 rate environment, whereas $52.6 \%$ worked in a low COVID-19 rate environment.

\section{OUTCOMES AND COVARIATES}

Demographic data were self-reported by participants. Measurement was focused on symptoms of anxiety, personality traits, peritraumatic dissociation, and posttraumatic stress for all participants. The psychological battery was composed of $n=4$ self-reports evaluating the anxiety (DASS-21), personality traits (BFI-10), and distress (IES-R and PDEQ) to measure the presence/absence of psychological symptoms and related severity.

Big Five Inventory-10 (BFI-10; Guido et al., 2015). The BFI-10 evaluates the five personality dimensions on a 5 -point scale ranging from 1 (strongly disagree) to 5 (strongly agree), each with two items: openness $(\mathrm{OP})$, conscientiousness (CO), emotional stability (ES), extraversion (EX), and agreeableness (AG).

Depression, Anxiety, and Stress Scale (DASS-21; Beaufort et al., 2017). The DASS is a clinical assessment that measures the three related states of depression, anxiety, and stress. It has 21 questions and takes about 3 minutes to complete. Each subscale measuring the emotional traits is composed of 7 items. We applied only the anxiety subscale.

Impact of Event Scale-Revised (IES-R; Weiss, 2007). It is a 22-item self-report questionnaire to measure the subjective response to a specific traumatic event, especially in the response sets of intrusion (intrusive thoughts, nightmares, intrusive feelings and imagery, dissociative-like re-experiencing), avoidance (numbing of responsiveness, avoidance of feelings, situations, and ideas), and hyperarousal (anger, irritability, hypervigilance, difficulty concentrating, heightened startle), as well as a total subjective stress IES-R score. Scores higher than 33 are of concern; the
Predictive risk factors for frontline nurses in a pandemic 
higher the score, the greater the concern for posttraumatic stress and associated health and well-being consequences.

Peritraumatic Dissociative Experiences Questionnaire (PDEQ; Marmar et al., 1997). It is a 10-item self-report questionnaire measuring peritraumatic dissociation. The PDEQ has well-established psychometric properties, with higher total scores indicating increased peritraumatic dissociation. A score above 15 is indicative of significant dissociation.

Jessica Ranieri, Federica Guerra, Dina Di Giacomo

\section{STATISTICAL ANALYSES}

The data analysis was performed using SPSS statistical software, with a fixed $\alpha$-value $\leq .05$. All demographic data were analyzed and presented as number $(n)$ and percentage (\%). Using the MANOVA test as appropriate, we compared emotional severity by demographic variables. Spearman rank-order correlation was used to examine correlations among anxiety, peritraumatic, post-traumatic stress, and psychological traits.

\section{RESULTS}

First, we analyzed the prevalence of emotional symptoms among nurses (Table 1).

A considerable part of the sample (77.3\%) showed anxiety: $10.5 \%$ extremely severe, $13.1 \%$ severe, $28.9 \%$ moderate, and $23.7 \%$ mild level (DASS-21 subscale Anxiety $>8$ [score range $0-21]$ ). Fifty-five percent of the sample evidenced significant peritraumatic dissociative experience (PDEQ score > 15 [score range 1-50]) and $52.6 \%$ of nurses showed a probable presence of post-traumatic stress (IES-R score $>33$ [score range $0-88$ ]) and $47.3 \%$ resulted in no stressed emotional condition.

For dimensions of personality status, the prevalence rates for each category were: $79 \%$ high level of conscientiousness (21\% moderate, no low level); $57.9 \%$ moderate level of emotional stability $(28.9 \%$ high and $13.1 \%$ low level); $50 \%$ moderate level of openness (39.4\% high, $10.5 \%$ low level); $44.7 \%$ moderate extroversion level (36.8\% high, $18.4 \%$ low level); $42.1 \%$ moderate level of agreeableness $(34.2 \%$ low, $23.6 \%$ high level).

Comparisons (MANOVA test) within the various demographic characteristics demonstrated few significant differences between groups in DASS-21, PDEQ, and IES-R scores. According to age groups (median value $=35$ years old), younger nurses showed higher anxiety (DASS-21) than the old group $(\eta=0.62$, $p=.020$ ). Marital status was significant: single nurses evidenced higher anxiety than married $(\eta=0.77$, $p=.012$ ), and single nurses reported a higher level of post-traumatic stress than married $(\eta=0.77, p=.012)$.
Correlation analysis (Spearman test) was performed among PDEQ, DASS-21, BFI-10, and IES-R. The results summarized in Table 2 confirm correlation between anxiety (DASS-21) and peritraumatic dissociation and posttraumatic stress; also anxiety is positively correlated with the agreeableness variable of the BFI-10 test.

\section{DISCUSSION AND CONCLUSIONS}

This observational study was focused on the anxiety symptoms that developed in nurses in the Italian acute COVID-19 outbreak; we wanted to investigate the anxiety as a risk factor for traumatic distress. A significant proportion of nurses developed anxiety symptoms (from extremely severe to mild level) related to peritraumatic dissociative experience and a probable sign for posttraumatic stress disorders. The agreeableness, personality dimension featured by positive feeling and trusting, was more relevant in our target.

Our findings were obtained in the acute Italian COVID-19 outbreak and measured and quantified the psychological response of nurses in terms of anxiety as an early reaction to emotional distress and high risk for posttraumatic stress disorders; the personality dimensions did not mediate the emotional distress or the probable risk for posttraumatic stress disorder: the impact of the pandemic event on the mental health of nurses was strong and unmanageable by themselves; individual resources did not help professionals to overcome the distress. Nurses appeared to be exposed to mental distress and needed help. Our findings provided details for protective and predictive risk factors as well as mental health issues of nurses dealing with the pandemic. Previous researchers (Rossi et al., 2020; Lai et al., 2020) conducted a mental screening in public health emergency and outlined the risk trend for health workers. Particularly, Zhang et al. (2020) observed the prevalence of risk factors in healthcare workers for somatization, anxiety, obsessive-compulsive signs as well insomnia: those factors favor allostatic overload by the clinimetric approach. Moreover, Bhagavathula et al. (2020) highlighted that the mental health of health workers can be impacted by the lack of knowledge about COVID-19 infection potentiality and even the widespread misinformation (social media dissemination). The authors argued about the important question that arises regarding how information was managed to help frontline health care workers in times of public health emergency by the improvement of the dissemination of scientific and authentic contents. The limitations of several studies are related to the detection method: many of them were based on online surveys by social media. Our study has confirmed and implemented findings by psychological 


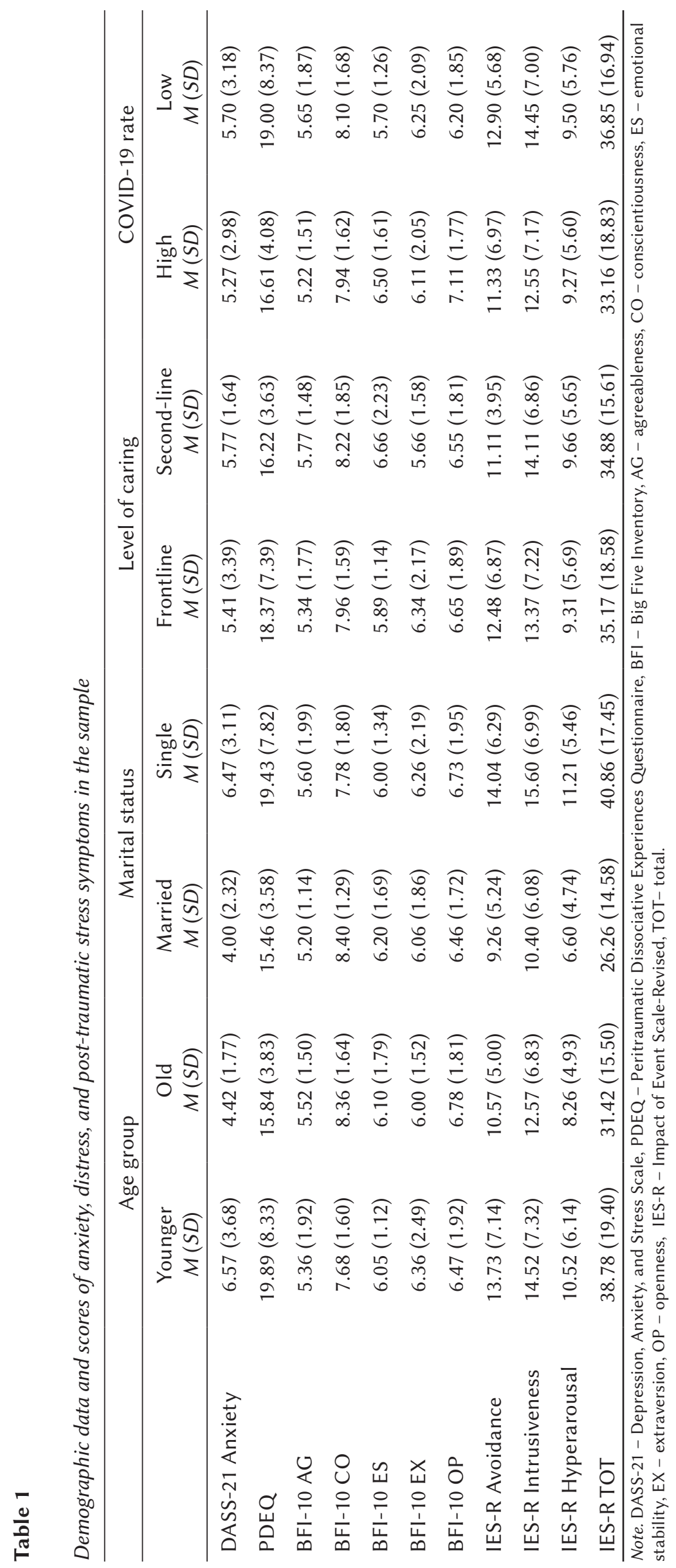

Predictive risk factors for frontline nurses in a pandemic 
Jessica Ranieri, Federica Guerra, Dina Di Giacomo

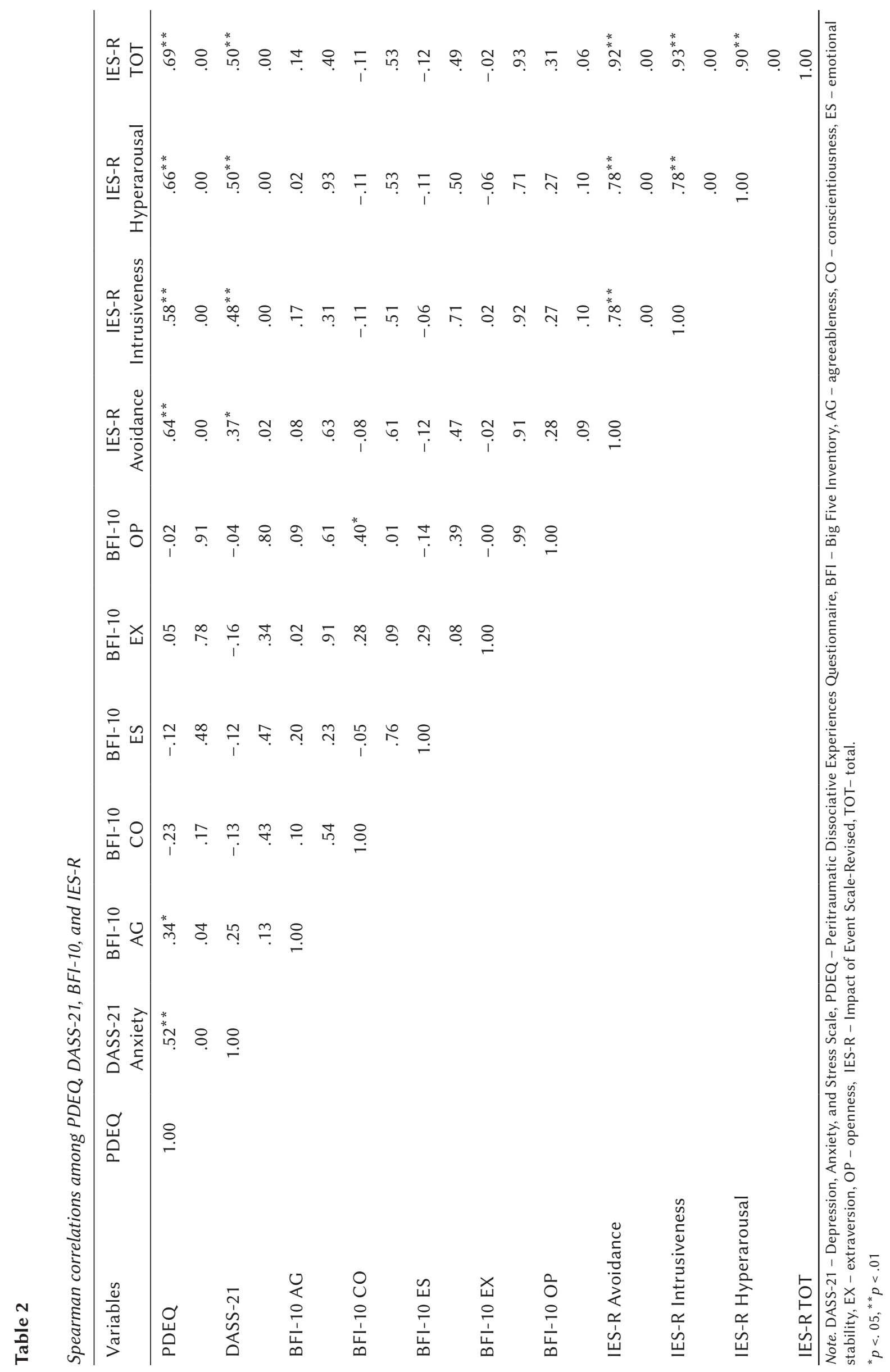


evaluation: the added value of our study is the effort to apply quantitative measurement toward formulation of a reliable scenario for future public health emergencies demanding a national mental health program in order a) to mitigate the negative effect of the current pandemic on the mental health of health workers, as well as b) to model healthcare policy for future public health emergencies.

In accordance with Greenberg et al. (2020), our findings evidenced the need to carry out mental health programs for health workers (especially nursing professionals) to prevent later mental disorders in those who take care of patients in the acute COVID-19 outbreak. Wellbeing psychological pathways tailored for nursing professionals could be a priority for the healthcare system to exploit the intensive coronavirus experience as valued-added and not as a mental risk for workers. The challenge is going to be to overcome and steer their mental fragility, turning it to a protecting process in a public health emergency. Greenberg et al.'s (2020) analysis evidenced the role played in psychological wellness of health workers a) to make impossible decisions and b) to work under extreme pressure. Both variables could cause moral injury and mental problems. Early support could mitigate the effect of the pandemic experience, reducing the risk for health workers' fragility.

The public health emergency is an extraordinary time and the lesson learnt was to act in order to arrange strategies toward the minimization of psychological risk for health workers, preventing long lasting damage to healthcare staff. Active monitoring should be the strategic action for the healthcare system in order to preserve and protect frontline workers. The challenge is to plan and implement a prevention program with evidence-based treatments.

\section{References}

Beaufort, I. N., De Weert-Van Oene, G. H., Buwalda, V. A. J., de Leeuw, J. R. J., \& Goudriaan, A. E. (2017). The Depression, Anxiety and Stress Scale (DASS-21) as a screener for depression in substance use disorder inpatients: a pilot study. $E u$ ropean Addiction Research, 23, 260-268. https:// doi.org/10.1159/000485182

Bhagavathula, A. S., Aldhaleei, W. A., Rahmani, J., Mahabadi, M. A., \& Bandari, D. K. (2020). Knowledge and perceptions of COVID-19 among health care workers: Cross-sectional study. JMIR Public Health and Surveillance, 6, e19160. https:// doi.org/10.2196/19160

Greenberg, N., Docherty, M., Gnanapragasam, S., \& Wessely, S. (2020). Managing mental health challenges faced by healthcare workers during covid-19 pandemic. British Medical Journal, 368, m1211. https://doi.org/10.1136/bmj.m1211
Guido, G., Peluso, A. M., Capestro, M., \& Miglietta, M. (2015). An Italian version of the 10-item Big Five Inventory: an application to hedonic and utilitarian shopping values. Personality and Individual Differences, 76, 135-140. https:// doi.org/10.1016/j.paid.2014.11.053

Lai, J., Ma, S., Wang, Y., Cai, A., Hu, J., Wei, N., Wu, J., Du, H., Chen, T., Li, R., Tan, H., Kang, L., Yao, L., Huang, M., Wang, H., Wang, G., Liu, Z., \& Hu, S. (2020). Factors associated with mental health outcomes among health care workers exposed to coronavirus disease 2019. JAMA Network Open, 3, e203976. https://doi.org/10.1001/jamanetworkopen.2020.3976

Marmar, C. R., Weiss, D. S., \& Metzler, T. J. (1997). The Peritraumatic Dissociative Experiences Questionnaire. In J. P. Wilson \& T. M. Keane (Eds.), Assessing psychological trauma and PTSD (pp. 412-428). Guildford Press.

Rossi, R., Socci, V., Pacitti, F., Di Lorenzo, G., Di Marco, A., Siracusano, A., \& Rossi, A. (2020). Mental health outcomes among frontline and second-line health care workers during the coronavirus disease 2019 (COVID-19) pandemic in Italy. JAMA Network Open, 3, e2010185. https:// doi.org/10.1001/jamanetworkopen.2020.10185

Weiss, D. S. (2007). The Impact of Event Scale: Revised. In J. P. Wilson \& C. S. Tang (Eds.), Crosscultural assessment of psychological trauma and PTSD (pp. 219-238). Springer.

Zhang, W. R., Wang, K., Yin, L., Zhao, W. F., Xue, Q., Peng, M., Min, B. Q., Tian, Q., Leng, H. X., Du, J. L., Chang, H., Yang, Y., Li, W., Shangguan, F. F., Yan, T. Y., Dong, H. Q., Han, Y., Wang, Y. P., Cosci, F., \& Wang, H. X. (2020). Mental health and psychosocial problems of medical health workers during the COVID-19 epidemic in China. Psychotherapy and Psychosomatics, 89, 242-250. https:// doi.org/10.1159/000507639
Predictive risk factors for frontline nurses in a pandemic 